\title{
Die neuen Herausgeber der Mitteilungen
}
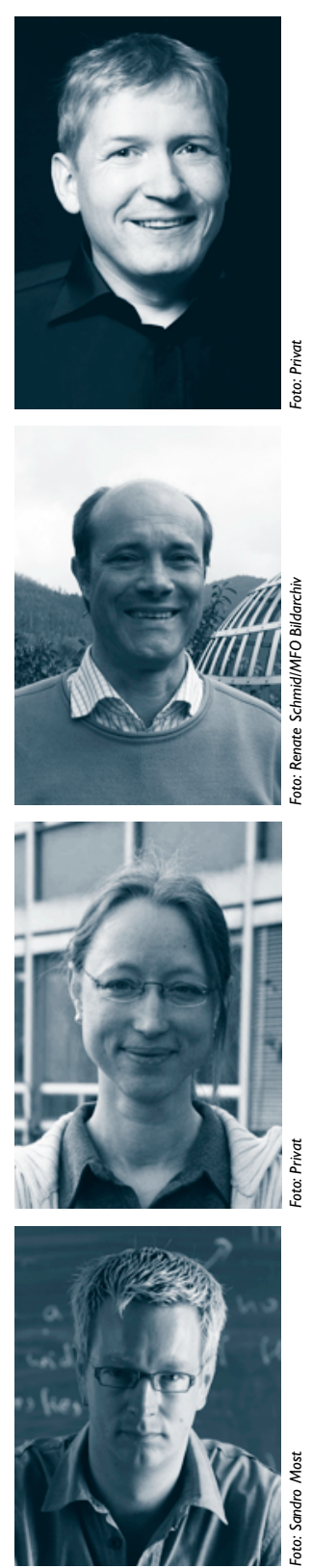

Martin Skutella, Günter Leugering, Brigitte Lutz-Westphal und Günter M. Ziegler (v. o. n. u.)
Martin Skutella wurde 1969 in Freiburg im Breisgau geboren. Er studierte Mathematik und Physik an der RWTH Aachen und wechselte danach an die TU Berlin, wo er 1998 promovierte. Nach einem Postdoc-Jahr in Belgien nahm er eine Gastprofessur an der Universität Bonn an und kehrte dann an die TU Berlin zurück. Nach einer weiteren Gastprofessur am Massachusetts Institute of Technology in Cambridge, USA, habilitierte er sich 2002 an der TU Berlin. Danach ging er an das Max-Planck-Institut für Informatik in Saarbrücken und wurde 2004 auf den Lehrstuhl für Diskrete Optimierung der Universität Dortmund berufen. Seit 2007 ist er Professor am Institut für Mathematik der TU Berlin und im DFG-Forschungszentrum MATHEON „Mathematik für Schlüsseltechnologien“. Sein Forschungsschwerpunkt liegt in der Kombinatorischen Optimierung, wo er sich unter anderem mit Netzwerkoptimierungsproblemen, Netzwerkflüssen und Scheduling beschäftigt.

Günter Leugering, 1953 in Mannheim geboren, studierte Mathematik mit Nebenfach Theoretische Physik in Frankfurt am Main, promovierte 1984 an der TU Darmstadt und habilitierte sich dort im Jahre 1988. Nach mehreren Auslandsaufenthalten und einer Gastprofessur am „Virginia Polytechnical Institute and State University“ Blacksburg VA, erhielt er 1989 einen Ruf auf eine tenure-track Professur an die Georgetown University in Washington DC und zur selben Zeit ein Heisenberg-Stipendium. Im Jahre 1992 nahm er einen Ruf auf eine C3Fiebiger-Professur an die Universität Bayreuth an. Nach einem Gastaufenthalt am Institut Henri Poincaré 1998 erhielt er im Jahre 1999 einen Ruf auf eine C4-Professur an die TU-Darmstadt. Er folgte schließlich im Jahre 2003 einem Ruf auf einen Lehrstuhl für Angewandte Mathematik an die Friedrich-Alexander-Universität ErlagenNürnberg. Seit 2008 leitet er das „Zentrum für Multiskalenmodellierung und Simulation“ im Exzellenzcluster „Engineering of Advanced Materials“. Sein Arbeitsgebiet ist Optimierung insbesondere im Kontext partieller Differentialgleichungen, Homogenisierung von Optimierungsproblemen und Steuerungstheorie.

Brigitte Lutz-Westphal, 197I in Tübingen geboren, studierte ab 199। Musik an der Hochschule der Künste Berlin und ab 1992 Mathematik an der Freien Universität Berlin. Das Studienjahr 1994/95 verbrachte sie in Paris an der Sorbonne und der Universität Paris Jussieu. I. Staatsexamen für das gymnasiale Lehramt 1998, Referendariat 1998-2000 in Tübingen und 2. Staatsexamen 2000. Danach freischaffende Tätigkeit als Musikerin in Berlin, Geburt des Sohnes Sebastian 2002. Ab 2002 Promotionsprojekt „Diskrete Mathematik für die Schule“ am Zuse-Institut Berlin, Promotion in Mathematikdidaktik 2006. 2004-2009 Mitglied im DFG-Forschungszentrum MATHEON „Mathematik für Schlüsseltechnologien“, dort u. a. Engagement in der Öffentlichkeitsarbeit und Nachwuchsförderung. 2006-2008 Vertretung einer Juniorprofessur an der Technischen Universität Berlin, seit 2008 Universitätsprofessorin für Mathematik und ihre Didaktik an der Hochschule Vechta. I I/2008 Ruf an die Freie Universität Berlin. Ihr Hauptinteresse liegt in der Entwicklung und Erprobung von Unterricht, Unterrichtsmaterialien und Lehrerfortbildungen zu modernen Anwendungen der Mathematik.

Günter M. Ziegler, Jahrgang 1963, ist geborener Münchner. Nach dem Studium an der LMU München promovierte er 1987 am MIT. Nach vier Jahren in Augsburg und einem Winter in Stockholm kam er 1992 ans ZIB Berlin. Seit Januar 1995 ist er Professor für Mathematik an der TU Berlin. Seine Forschung beschäftigt sich mit Diskreter Geometrie, besonders der Theorie der Polyeder, topologischen Methoden in der Kombinatorik sowie mit Problemen der Optimierung. Mit Martin Aigner schrieb er „Das BUCH der Beweise“, das inzwischen in 13 Sprachen vorliegt. 1997-2000 war er Herausgeber der ,Mitteilungen der DMV“, seitdem ist er Mitherausgeber und Kolumnist (,Mathematik im Alltag"). 2006-2008 war er der Präsident der DMV und in dieser Funktion ein Initiator des Jahres der Mathematik 2008. Im Auftrag des DMV-Präsidiums leitet er seit 2009 das Medienbüro der DMV (s. S. 50). 